\title{
Ultrasound-guided midline catheter insertion in a quarantined COVID-19 head trauma patient
}

\author{
Seok Hwa Youn, Sun Young Baek, Jiho Yoon, Younghwan Kim \\ Department of Surgery, National Medical Center, Seoul, Korea
}

Received April 23, 2020

Revised June 22, 2020

Accepted June 22, 2020

Correspondence to

Younghwan Kim

Department of Surgery, National

Medical Center, 245 Eulji-ro, Jung-gu,

Seoul 04564, Korea

Tel: $+82-2-2260-7114$

Fax: +82-2-2267-8685

E-mail: galgunbam2@daum.net

\begin{abstract}
A midline catheter offers various benefits as compared to both a central venous catheter and a peripheral intravenous catheter. A 55-year-old man with COVID-19 was transferred to our center for treatment of head trauma. He required long-term intravenous therapy, but peripheral insertion catheter introduction was too difficult. Instead, a midline catheter was inserted under ultrasonography guidance in the special environment such as isolated intensive care unit which had limited space and transfer to other place due to contagious disease.
\end{abstract}

Key Words: Vascular access devices, COVID-19, Trauma

\section{Introduction}

The central venous catheter (CVC) has been used for the specific indication of total parenteral nutrition, special medications which require CVC, and the introduction of high concentration electrolytes, for example. In addition, they are preferred for the purpose of alleviating the patient's discomfort and pain for numerous needle insertions during medium- to long-term intravenous therapy (1). However, they should be used cautiously, understanding the risk of the potentially fatal complications such as systemic infection, pneumothorax, and central venous thrombosis. A midline catheter (MC), recently introduced to Korea, is an intravenous catheter that is inserted through an upper arm vein under ultrasonography guidance. We can consider insertion of MC which has similar role to a peripherally inserted central catheter (PICC), that can replace $\mathrm{CVC}$ with reducing the risk of complications if there is no mandatory indication of CVC (2). The authors report their experience of insertion the $\mathrm{MC}$ to the isolated patient with head trauma who was diagnosed with coronavirus disease 2019 (COVID-19) in intensive care unit (ICU).

\section{Case presentation}

A 55-year-old man diagnosed with COVID-19 was transferred to our trauma center after a fall from his bed, suffering a skull fracture, a subdural hematoma, a traumatic subarachnoid hemorrhage, and a large hemorrhagic contusion on his left frontal lobe. He was treated in a special ICU for COVID-19 patients. His peripheral veins did not permit insertion of the peripheral intravenous (PIV) line, despite multiple attempts, all unsuccessful. Although he was fine adjusted for continuous tubal feeding for his daily nutritional needs, he still required IV lines for medi-

(c)This is an Open Access article distributed under the terms of the Creative Commons Attribution Non-Commercial License (http://creativecommons.org/licenses/by-nc/4.0) which permits unrestricted noncommercial use, distribution, and reproduction in any medium, provided the original work is properly cited.

Copyright (c) 2020 Korean Association for Research, Procedures and Education on Trauma. All rights reserved. 
cations. Although we could have inserted a PICC or CVC, as would be common, we decided to use the MC, having been recently introduced to our country and offering several benefits.

The process of insertion:

- Level D personal protection equipment was applied. A maximal sterile barrier was applied because there was a possibility of contamination because level D could not adhere to the body (Fig. 1).

- $8 \mathrm{~cm}$ of 18 gage catheter made by PowerGlide Pro ${ }^{\mathrm{TM}}$ (MC, Bard Access Systems, Inc., 605 North 5600 West, Salt Lake City, Utah USA 84116) was provided (Fig. 2).

- Lidocaine was injected for regional anesthesia, and tourniquet was not applied.

- After an ultrasound-guided puncture was made in left basilic vein with MC, the guidewire in the hand piece was simply pushed and the hand piece was removed.

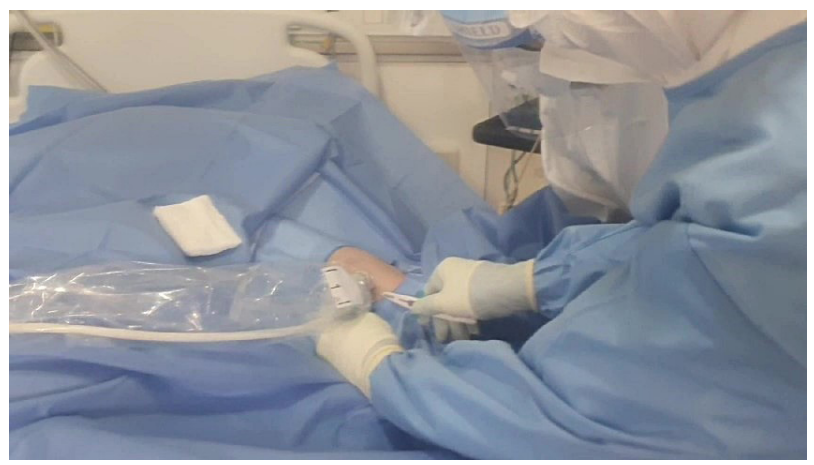

Fig. 1. Maximal sterile barrier to avoid contamination with minimal manpower in a limited space.

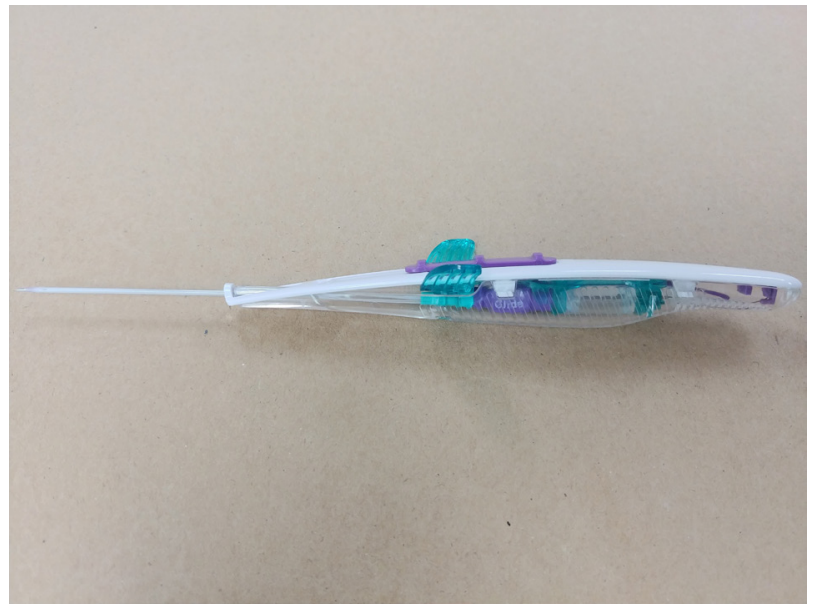

Fig. 2. PowerGlide Pro ${ }^{\mathrm{TM}}$.
- The plug with handle was removed and checked venous blood out.

Total time for the procedure, excluding draping, was 3 minutes and 40 seconds, using a single puncture; there were no complications such as hematoma or venous rupture.

\section{Discussion}

The MC is usually inserted between 1.5 inches above and below the antecubital fossa into the basilic, cephalic or brachial vein. The tip of the catheter is placed at approximately the axilla, so that its position allows for further hemodilution of medication, decreasing any associated phlebitis, infiltration and discomfort (2). This catheter can remain in situ for up to 28 days without complications, and it can endure the osmolarity $<600 \mathrm{mOsm}, \mathrm{pH}$ of $5-9$ of an infusion solution or blood products. It also provides a path for contrast introduction for computed tomography due to its high flow tolerability $(3,4)$.

Except in certain mandatory indications for CVC (e.g., $\mathrm{TPN}, \mathrm{CaCl}$ ), MC reduces complications such as local infection, thrombosis, central line-associated bloodstream infection, pneumothorax, and life-threatening hemorrhage. Additionally, MC use can be almost free from CVC-specific complications such as failure to place the catheter, arterial puncture, subcutaneous hemorrhage, hemothorax and cardiac arrest $(5,6)$.

MC also can be compared to PIV, offering better results with regard to such complications as catheter infection, thrombosis, infiltration, dislodgement, and thrombophlebitis. Additionally, some studies reported much lower failure rates for the first insertion attempt (PIV:MC, 26\%:3.2\%)(7,8).

For cases using PICC, the patient needs to transfer to an angiography facility or at the bedside in a large enough room and with sufficient personnel to handle the long guidewire. Both are difficult situations to arrange in an isolated environment for contagious diseases. MC can be inserted with minimal manpower in a limited space versus PICC usage(Fig. 1).

With the aforementioned benefits, the indications for $\mathrm{MC}$ insertion are prolonged intravenous infusion and laboratory drawn, and difficult venous access requiring multiple attempts at peripheral intravenous line placement which also can apply to trauma patient. In situations 
involving isolated or quarantined patients, such as those with COVID-19, who may have limited access to certain facilities for examinations and procedures, MC usage can provide an opportunity for a bedside procedure with minimal manpower and space, increasing CVC free day while decreasing the potential for various complications of CVC $(5,9)$.

\section{Conflict of interest}

No potential conflict of interest relevant to this article was reported.

\section{References}

1. Bundgaard Madsen E, Sloth E, Skov Illum B, Juhl-Olsen $\mathrm{P}$. The clinical performance of midline catheters-an observational study. Acta Anaesthesiol Scand. 2020;64(3):394-9.

2. Rosenthal K. Bridging the I.V. access gap with midline catheters. Nursing. 2008;38 Suppl Med:2, 4-5.

3. Alexandrou E, Ramjan LM, Spencer T, Frost SA, Salamonson Y, Davidson PM, et al. The use of midline catheters in the adult acute care setting-clinical implications and recommendations for practice. Journal of the Association for Vascular Access. 2011;16(1):35-41.

4. Carrero MC, Montealegre MS, Cubero MP. Medial venous catheter or midline (MVC). Revista de enfermeria (Barcelona, Spain). 2014;37(1):36-41.

5. Adams DZ, Little A, Vinsant C, Khandelwal S. The midline catheter: a clinical review. The Journal of Emergency Medicine. 2016;51(3):252-8.

6. Caparas JV, Hu JP. Safe administration of vancomycin through a novel midline catheter: a randomized, prospective clinical trial. J Vasc Access. 2014;15(4):251-6.

7. Helm RE, Klausner JD, Klemperer JD, Flint LM, Huang E. Accepted but unacceptable: peripheral IV catheter failure. J Infus Nurs. 2015;38(3):189-203.

8. Moureau N, Sigl G, Hill M. How to establish an effective midline program: a case study of 2 hospitals. Journal of the Association for Vascular Access. 2015;20(3):179-88.

9. Griffiths V. Midline catheters: indications, complications and maintenance. Nursing Standard (through 2013). 2007;22(11):48. 